\title{
FIB-Nanotomography of Particulate Systems_-Part I: Particle Shape and Topology of Interfaces
}

\author{
Lorenz Holzer, ${ }^{\dagger}$ Beat Muench, Markus Wegmann, and Philippe Gasser \\ Empa Materials Science and Technology, 3D-Mat, CH-8600 Dübendorf, Switzerland
}

Robert J. Flatt

Sika Technology AG, Corporate Research \& Analytic, CH-8048 Zürich, Switzerland

\begin{abstract}
A new 3D-microscopy method, focused ion beam-nanotomography (FIB-nt), has been applied to the statistical particle shape analysis and for topological characterization of granular textures in cement samples. Because of its high resolution (15 $\mathrm{nm})$, FIB-nt reveals precise microstructural information at the submicrometer scale, which cannot be obtained with conventional tomography methods. It is demonstrated that even from complex granular textures with dense agglomerates, it is possible to identify the individual sub-grains. This is the basis for reliable statistical shape analysis. For this purpose, moments of inertia were determined for particles from five different grain size fractions of a given cement, which provides important input data for future modeling of rheology and hydration processes. In addition, FIB-nt was used for topological characterization of the particle-particle interfaces in the dense and fine-grained granular textures. The unique 3D-data obtained with FIB-nt thus open new possibilities for quantitative microstructure analysis and the data can be used as structural input for object-oriented modeling.
\end{abstract}

\section{Introduction}

$\mathrm{M}$ ANY materials science disciplines such as concrete technology, ceramics, metallurgy, and colloid science are dealing with the micromechanical behavior of particulate systems and with the optimization of the corresponding material properties. An increasing number of investigations aim for a theoretical and/or numerical treatment of these systems, e.g., by modeling processes such as granular flow by discrete element methods. ${ }^{1,2}$ For example, a promising approach to model viscous flow in cement pastes is based on dissipative particle dynamics (DPD). ${ }^{3}$ So far, this method has allowed to follow the motion of particles in a sheared liquid medium and to determine, in the absence of surface forces among the particles, the plastic viscosity of suspensions as a function of particle volume fraction and particle size distribution (PSD). In contrast to many other object-oriented models, these DPD investigations allow to handle particles of any desired shape rather than only idealized geometries as spheres or ellipsoids.

Thus, computational models are reaching the stage where they will allow simulation of the role of shape in the complex behavior of granular medium. It is recognized that a range of properties are affected by shape, as illustrated in Table I. ${ }^{4-6}$ However, the quality of these simulations will depend not only on how well physical laws dictating particle interactions can be

R. Riman-contributing editor

\footnotetext{
Manuscript No. 21061. Received October 10, 2005; approved January 27, 2006.

This work was financially supported by Sika technology AG, Corporate Research and Analytic, Switzerland

${ }^{\dagger}$ Author to whom correspondence should be addressed. e-mail: lorenz.holzer@empa.ch
}

implemented but also on the quality of the three dimensional (3D) shape descriptions they will use.

This is of particular concern for particulate systems in which the fine fraction plays a significant role in macroscopic properties (reactivity, agglomeration, etc.). Indeed, X-ray tomography, which might appear as the prime method for 3D shape characterization, provides a voxel resolution of approximately 0.5 to 1 $\mu \mathrm{m}$ and consequently only the morphology of particles larger than $15 \mu \mathrm{m}$ can be reconstructed with this technique. ${ }^{7}$ This is clearly insufficient to handle agglomerating systems as well as reactive ones such as portland cement (PC), in which particles below $10 \mu \mathrm{m}$ represent about $90 \%$ of the specific surface.

As an alternative to X-ray tomography, 3D microscopy serial sectioning has been used, whereby the microstructural reconstruction is based on stacks of successive images acquired from depth profiles after mechanical polishing. In such investigations, the resolution of mechanical serial sectioning techniques is limited by the precision and thickness of the layer-by-layer erosion, which is in the micrometer range. As the spacing between the images is usually much larger than the pixel resolution of the images in the stack, object recognition must be carried out first in two dimensional (2D) space. The subsequent 3D-reconstruction is then based on a correlation of the identified $2 \mathrm{D}$ objects into the third direction. Thus, in contrast to non-destructive $\mathrm{X}$ ray tomography techniques, it is hardly possible to obtain a voxel-based data volume with conventional serial sectioning because of the coarse interlayer spacing.

Advanced serial sectioning with much smaller interlayer spacings can be achieved by means of ion milling with the focused ion beam (FIB) technique. The so-called FIB tomography method was introduced for the reconstruction of submicrometersized particles within FeAl-based nanocomposites, whereby an interlayer spacing in the range of $100 \mathrm{~nm}$ could be reached. ${ }^{8,9}$ However, with the single-beam FIB technique, the stage has to be tilted and repositioned between each in-plane erosion and out-of-plane imaging step. Thereby, the mechanical tilting of the stage induces imprecisions that limit the resolution and reproducibility of the interlayer spacings. As a result, the data are not suitable for quantification of microstructural features at the submicrometer scale.

To some extent, this limitation has been overcome with the more recent dual-beam FIB machines, which consist of an ion column for milling and an electron column for SEM imaging. Such machines, which make stage tilting and repositioning during the serial sectioning obsolete, bring erosion thickness down to below $100 \mathrm{~nm}$ in automated serial sectioning procedures. ${ }^{10}$ However, the alternation of milling and imaging is very time consuming and over such long acquisition times instabilities of sample, stage, electronics of the optical columns induce remarkable shift components that compromise the data quality for erosion thickness below $100 \mathrm{~nm}$. Hence, without automated drift-correction procedures, the data from high-resolution FIB serial sectioning are affected by uncontrolled drift sources that 
Table I. Classification and Phenomenology of Particulate Systems (Modified after Scott ${ }^{4}$ )

$\begin{gathered}\text { Individual particles and } \\ \text { their surfaces }\end{gathered}$
Increasing complexity
of particle systems

\begin{tabular}{|c|c|c|c|c|}
\hline Scale(s) & Nano $->$ micro & Nano-meso-micro & (Sub-)micro-macro & nano-micro-macro \\
\hline $\begin{array}{l}\text { Topological } \\
\text { features and } \\
\text { parameters }\end{array}$ & $\begin{array}{l}\text { Particle size } \\
\text { Particle shape/ } \\
\text { moments of inertia } \\
\text { Surface topogr. } \\
\text { Conformation of } \\
\text { adsorbed surfactant }\end{array}$ & $\begin{array}{l}\text { Agglomerates } \\
\text { Fragments } \\
\text { Interface between } \\
\text { the primary particles } \\
\text { Contact normal } \\
\text { vector and contact } \\
\text { curvature }\end{array}$ & $\begin{array}{l}\text { Particle packing in dry } \\
\text { powders }+ \text { size } \\
\text { distribution (PSD) } \\
\text { Particle structures } \\
\text { in suspensions } \\
\text { No. of contacts } \\
\text { Percolation threshold }\end{array}$ & $\begin{array}{l}\text { Topology of solid granular } \\
\text { textures (ceramics, mortar, } \\
\text { concrete, metals) } \\
\text { PSD and grain shape } \\
\text { Topology of cracks and } \\
\text { or porosity }\end{array}$ \\
\hline
\end{tabular}

Examples of properties and processes

$\begin{array}{ll}\text { Surface properties, } & \text { Contact mechanics } \\ \text { chemical potentials } & \text { Particle interaction and } \\ \text { Adsorption, } & \text { interpart. forces } \\ \text { Desorption, } & \text { Agglomeration, } \\ \text { Topotactic crystal } & \text { attraction/repulsion, } \\ \text { growth, Dissolution } & \text { fragmentation }\end{array}$

Surface properties, Desorption, Topotactic crystal fragmentation

Granular mechanics
Flow properties
Particle packing
Network formation
of connected particles
and restructuring

Fracture mechanics Continuum properties such as strength, elasticity, optical density, conductiv. Grain boundary migration during sintering Crack propagation

\begin{tabular}{|c|c|c|c|c|}
\hline $\begin{array}{l}\text { Examples of } \\
\text { analytical } \\
\text { techniques }\end{array}$ & $\begin{array}{l}\text { Laser diffraction } \\
\text { for particle size } \\
\text { distribution (PSD) } \\
\text { AFM for surface } \\
\text { topography } \\
\text { TOF-SIMS or auger } \\
\text { spectroscopy f. } \\
\text { surface chemistry }\end{array}$ & $\begin{array}{l}\text { FIB-tomography }{ }^{\dagger} \text { for } \\
\text { topology of } \\
\text { agglomerates } \\
\text { AFM for force } \\
\text { measurements }\end{array}$ & $\begin{array}{l}\text { Rheometry for viscosity } \\
\text { measurement } \\
\text { (macroscopic) } \\
\text { FIB-tomography }{ }^{\dagger} \text { for } \\
\text { topological characteri- } \\
\text { zation of particle } \\
\text { packing at submicro- } \\
\text { meter scale }\end{array}$ & $\begin{array}{l}\text { Macroscopic tests of } \\
\text { mechanical, electrical, } \\
\text { optical, thermal, and } \\
\text { chemical properties } \\
\text { Microscopic characteri- } \\
\text { zation of granular } \\
\text { textures, eg. with FIB- } \\
\text { nanotomography. }\end{array}$ \\
\hline
\end{tabular}

${ }^{\dagger}$ Used in this study. PSD, particle size distribution.

reduce the precision and the reproducibility of the final quantitative microstructure analyses.

In this paper, we overcome these limitations with an improved automated serial sectioning procedure that we have developed for dual-beam FIB with integrated drift correction for each slicing and imaging cycle. ${ }^{11}$ With this method, called FIBnanotomography (nt), a reproducible spacing of $15 \mathrm{~nm}$ can be achieved. As the slicing distance has a similar length scale as the pixel resolution in the imaging plane, the entire stack of images can be transformed directly into a voxel-based data volume, and all image analysis procedures can be performed directly in $3 \mathrm{D}$ space. FIB-nt is thus capable of providing unique and accurate $3 \mathrm{D}$ data at resolutions of $15 \mathrm{~nm}$, which, at the moment, can neither be achieved with X-ray tomography nor with conventional FIB tomography or with mechanical serial sectioning methods.

In this study, we have applied FIB-nt to characterize PC. High-resolution 3D data of cement particles are of particular interest, as the predictability of the properties of cementitious materials is currently unsatisfactory and thereby the use of idealized particle shapes is often listed as one limitation of existing models. In terms of rheology, the role of fine particles in agglomeration and shape in plastic viscosity as well as the number of contacts is certainly essential. In terms of reactivity, $90 \%$ of the surface area is accounted for by particles smaller than 10 microns, whereas the corresponding volume fraction is only about $40 \%$ (as determined for the cement used in this study with laser granulometry). Thus, predictive models for properties of cementitious materials can certainly benefit from enhanced quality of $3 \mathrm{D}$ imaging that FIB-nt can deliver. Various other advantages of the FIB-nt method are also illustrated in the paper as for instance the ability to identify internal fractures and corresponding subgrains from larger, fragmented particles or the ability to characterize the topology of particle-particle interfaces.

\section{Materials and Sample Preparation}

FIB-nt analyses were performed with different grain size fractions of an ordinary portland cement (PC) (OPC CEM I 32.5R, Lafarge Cement UK, Oxon, UK). The grain size fractions were separated from the dry cement powder by air classification (Alpine MRZ2, Hosokawa, ALPINE AG, Augsburg, Germany). For each fraction, $10 \%, 50 \%$, and $90 \%$ fractiles, as obtained by laser diffractometry (Malvern Zetasizer 4, Worcestershire, UK), are given in Table II ( 5 min ultrasonic treatment in ethanol). The samples for FIB analysis were prepared by compacting the dry powders (fractions 1 to 5 ) at moderate pressures in a small cylinder ( $5 \mathrm{~mm}$ diameter, $10 \mathrm{~mm}$ height). Subsequently, the cylindrical samples were impregnated with Wood's metal (Bi $50 \%$, $\mathrm{Pb} 25 \%$, Sn $12.5 \%$, Cd 12.5\%; melting point $70^{\circ} \mathrm{C}$ ). Metal impregnation was performed in order to increase the imaging contrast, which reduces segmentation problems during data processing. ${ }^{12}$ For impregnation, the samples were placed in a bed of Wood's metal granules in a vacuum container and heated up to $85^{\circ} \mathrm{C}$. After heating and melting of the metal, pressures up to $3 \mathrm{kbars}$ were applied in order to fill the pores with the metal. The subsequent grinding and polishing steps were identical to those used when preparing SEM samples. ${ }^{13}$ Finally, the samples were glued onto an aluminum stub and a Pt layer was deposited 
Table II. Particle Size Analyses with Laser Diffractometry

\begin{tabular}{lcccc}
\hline $\begin{array}{l}\text { Grain size } \\
\text { fraction }\end{array}$ & $\begin{array}{c}\text { Rotation velocity } \\
\left(\times 10^{3}\right)\end{array}$ & $\begin{array}{c}10 \% \text { fractile } \\
(\mu \mathrm{m})\end{array}$ & $\begin{array}{c}50 \% \text { fractile } \\
(\mu \mathrm{m})\end{array}$ & $\begin{array}{c}90 \% \text { fractile } \\
(\mu \mathrm{m})\end{array}$ \\
\hline Bulk OPC & - & 2.16 & 19.35 & 73.58 \\
1 & $>20$ & 0.32 & 0.91 & 2.41 \\
2 & $15-20$ & 0.37 & 2.1 & 4.63 \\
3 & $10-15$ & 0.58 & 4.76 & 8.79 \\
4 & $5-10$ & 0.86 & 8.63 & 15.02 \\
5 & $3.5-5$ & 7.44 & 14.16 & 23.97 \\
6 & $2.5-3.5$ & 12.58 & 21.36 & 34.01 \\
7 & $2.2-2.5$ & 18.39 & 30.52 & 45.54 \\
8 & $<2.2$ & 32.51 & 54.25 & 89.17
\end{tabular}

Eight grain size fractions of cement powder were separated by air classification from an ordinary portland cement. Fractions 1-5 were analyzed with FIB-nt. ${ }^{\dagger}$ Rotation velocity for air classification. FIB-nt, focused ion beam-nanotomography.

by sputtering. In order to avoid mechanical stage drift during the FIB slicing procedure, the sample dimensions were kept as small as possible (edge lengths of a few millimeters).

As the samples for 3D analysis represent grain-supported textures, they include geometrical information about the spatial distribution of particles in a solid material, i.e., about the entire granular texture. The quality of such 3D data thus goes far beyond the counting statistics of conventional particle analysis methods such as light scattering of suspensions (e.g., laser diffractometry) and projections of particles on filtrates (e.g., 2D microscopy with SEM or TEM). For the future, it is intended to use this kind of information to study particle interactions in suspensions by stabilizing the microstructures with cryo techniques.

\section{Three-Dimensional Particle Analysis with FIB-nt}

As mentioned in the introduction, FIB-nt provides the means to perform high-resolution $3 \mathrm{D}$ imaging of samples on and above the $\mu \mathrm{m}$ scale. The specialty of the FIB-nt method is the combination of drift-corrected high-resolution 3D imaging (voxel resolution $<20 \mathrm{~nm}$ ) with the ability to analyze relatively thick samples above the $\mu \mathrm{m}$ scale. This provides new possibilities for quantitative characterization of fine-grained microstructures, such as networked granular textures and pore structures. In the following sections, the FIB-nt procedure is briefly summarized, with a special focus on its application to particle analysis. A more general description of the FIB-nt method has been given in a previous publication. ${ }^{11}$ The procedure was implemented on a dual-beam FIB (FEI Strata DB235) using the AutoScript language for FEI workstations. ${ }^{14}$

\section{(1) Principles of Image Acquisition with FIB-nt and Subsequent 3D Reconstruction}

The FIB-nt procedure includes the following steps: (a) preparation of a cube of suitable size, (b) application of reference marks and calibration of ion-beam shift $(\mathrm{V} / \mu \mathrm{m})$ for automated drift correction, (c) serial sectioning and acquisition of a stack with hundreds of images including drift correction after each cycle of imaging and erosion, and (d) off-line data processing (alignment of image stack, 3D reconstruction, segmentation, visualization, and quantification).

The geometrical relationships for cube preparation (a) and serial sectioning (c) are shown in Fig. 1. The sample was placed at the eucentric point where the ion beam and the electron beam converged at an angle of $52^{\circ}$. The ion beam (y-direction) was used to prepare the imaging planes ( $x-y$ sections), while the images were acquired with the scanning electron column. During the automated serial sectioning procedure, thin layers of the $x-y$ planes were eroded with the ion beam and imaged with the electron beam in an alternating process. In this way, a stack with

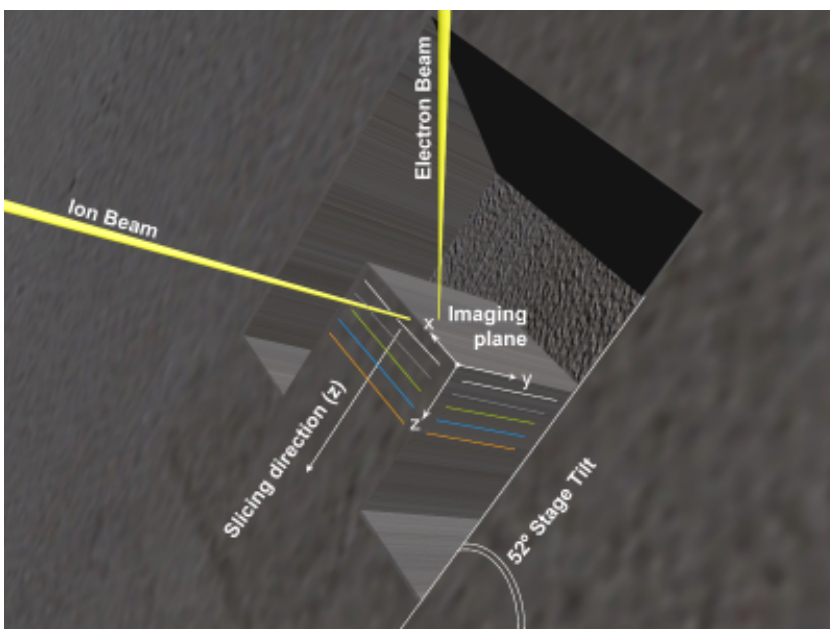

Fig. 1. Schematic illustration of the focused ion beam-nanotomography (FIB-nt) serial sectioning procedure with dual-beam FIB.

hundreds of images was produced, while moving through the sample in the z-direction.

A major challenge for the serial sectioning method is achieving a reproducible spacing between the individual images. Here, the thickness of the layers should be of the same magnitude as the pixel resolution of the SEM images (i.e., a few $\mathrm{nm}$ ) so that the stack of $2 \mathrm{D}$ images can be transformed into a voxel-based data volume and subsequent computational analysis (segmentation and quantification) can be performed directly in 3D space. As the duration of the slicing procedure spans over several hours, uncorrected beam, stage, and sample drift in the $\mathrm{z}$ direction can greatly affect the reproducibility of FIB-nt results. For this reason, an automated drift-correction procedure is implemented. Drift components in the $x$ and $z$ directions are measured after each slicing and imaging loop. For this purpose, reference marks on the sample surface ( $x-z$ plane) are localized by means of automated pattern recognition. The position of the ion beam is then corrected according to the measured displacement. The remaining drift components of the electron beam in the $x$ and $y$-directions are corrected during off-line data processing by applying least square fitting algorithms to achieve alignment of the images in the stack.

As an illustration of the raw data, a selection of 10 SEM images from grain size fraction 3 with a $z$-spacing of $60 \mathrm{~nm}$ is shown in Fig. 2. The imaging planes ( $x-y$ sections) exhibited a grain-supported texture of the cement powder. This example documents the slow but distinct changes that occur over a displacement of $600 \mathrm{~nm}$ in the z-direction. For 3D reconstruction of the microstructure, a specific region of interest (ROI) was selected from these raw data images, as indicated by the black frame in Fig. 2, slice no. 150.

The subsequent off-line data processing of the image stacks included preprocessing (background leveling and alignment), segmentation, quantification, and visualization. For particle analysis based on 3D data volumes, special attention must be given to the accurate identification of individual particles, which is a segmentation problem, and to the stereological correction for particle truncation at the intersection with the cube boundaries. Specific segmentation techniques for particle identification have been developed during this study. The computational methods for object recognition and stereological correction of the truncation effects at the cube boundaries are described in more detail in a separate publication-see part II. ${ }^{15}$

All algorithms were programmed in the working environment of Matlab 7.0, and they operated in 3D space. For fast inspection and visualization of the results, Amira 3.1 software was used. Examples of the resulting 3D representations are shown in Fig. 3: after preprocessing (upper cube), after segmentation 


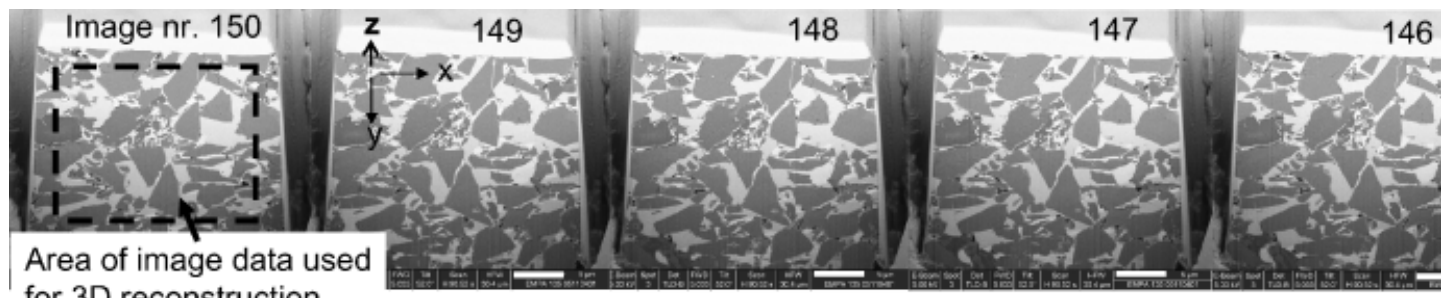
for $3 \mathrm{D}$ reconstruction

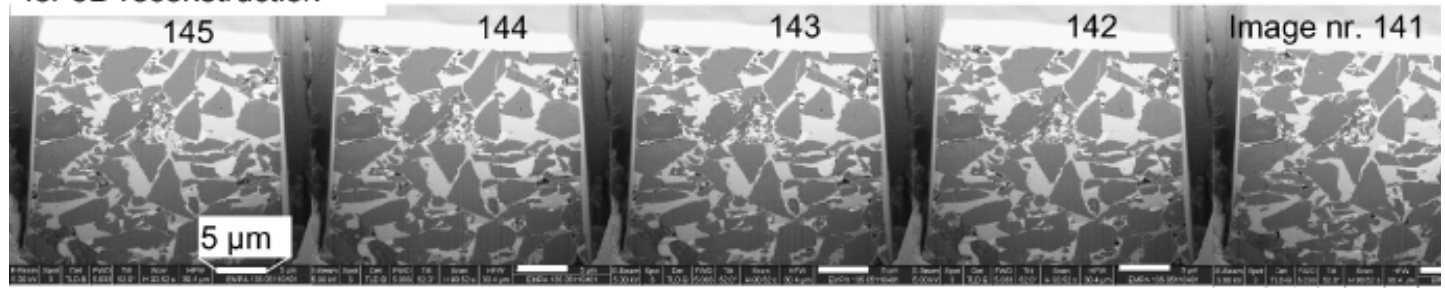

Fig. 2. Selection of 10 consecutive electron images (slices no. 141 to 150) from the raw data stack of cement fraction 3 (slicing distance: $60 \mathrm{~nm}$ ). The cement grains appear dark gray, and the matrix in light gray represents porosity, which has been filled with Wood's metal.

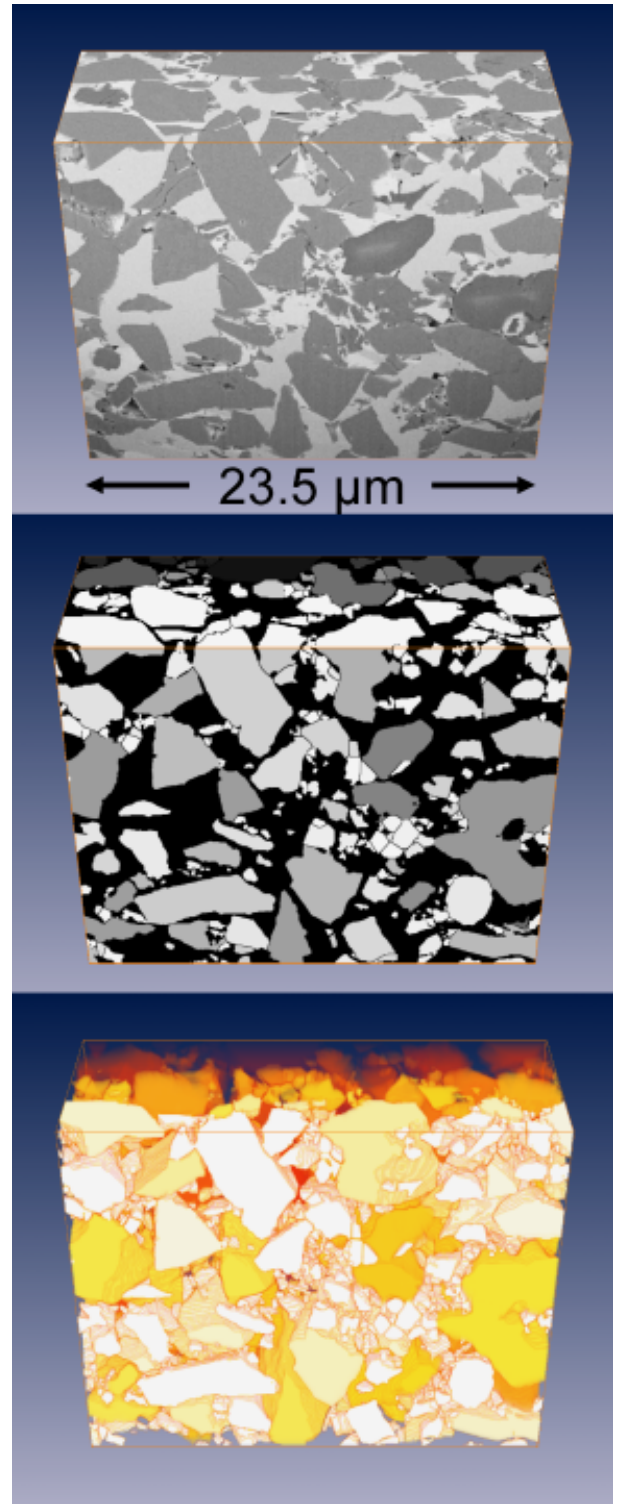

Fig. 3. Three dimension (3D) reconstructions of image stacks from grain size fraction 3: Upper cube - raw data after alignment and preprocessing. Middle cube - after segmentation. Bottom cube - semitransparent 3D visualization, each particle is labeled with a specific color value. Cube size: $23.5 \times 19.2 \times 9.5 \mu \mathrm{m} /$ voxel resolution: $30 \times 38 \times 60 \mathrm{~nm} /$ no. of particles: 2236 (total), 1404 (inside). (middle cube), and as a semitransparent visualization with color labeling of the different objects (bottom cube). This example shows well how, in contrast to conventional X-ray tomography, FIB-nt allows the morphology of particles below the micrometer scale to be resolved.

\section{(2) Specific Imaging Problems for 3D Particle Analysis: Representativity versus Resolution}

A major problem for particle analysis with $3 \mathrm{D}$ microscopy is the inherent conflict between resolution and representativity. ${ }^{16-18}$ The ideal resolution depends mainly on the average particle size, although the resolution should be high enough to also characterize accurately the smallest objects of interest. In addition, a large number of particles should be enclosed within the data volume in order to yield statistically representative analyses. Consequently, relatively large data volumes with a high voxel resolution would be ideal for quantitative $3 \mathrm{D}$ microscopy. However, this results in a large voxel matrix that can easily exceed the capabilities of FIB-nt. The limitations of FIB are given by the pixel matrix of the SEM images $(1024 \times 884)$ and by the acquisition time for serial sectioning (a maximum of 300 images can be acquired during one FIB session). Consequently, for particle analysis with FIB-nt, voxel matrix, voxel dimensions (magnification), and the size of the analyzed volume must be optimized according to particle size and heterogeneity of each sample and also according to the technical limitations of FIB.

Figure 4 shows the dimensions of the analyzed volumes for grain size fractions 1 through 5 . The cubes represent the regions of interest, which were cropped from the raw data for $3 \mathrm{D}$ reconstruction and particle analysis. This was the first time that FIB-nt was applied over such a wide range of magnifications. The size of the analyzed volumes ranges from $81 \mu \mathrm{m}^{3}$ for sample 1 (voxel resolution $12 \mathrm{~nm}$ ) up to more than $100000 \mu^{3}$ for sample 5 (voxel resolution $116 \mathrm{~nm}$ ). More detailed data regarding the voxel dimensions and the voxel matrix are given in Table III.

\section{Results and Discussion}

The reconstructed granular textures of the five image stacks from FIB-nt (Table III) are shown as a 3D scale model in Fig. 5. Between 1200 and 3800 individual particles were present within each of the analyzed cubes. The particle size ranged from 100 $\mathrm{nm}$ up to $50 \mu \mathrm{m}$. For statistical analysis based on microscopic data, it is crucial that each particle is recognized as a single object. However, like many other fine-grained granular materials, cement powders exhibit very heterogeneous and irregular particle structures. The particles are affected by fragmentation and they tend to form agglomerates. Consequently, the identification 


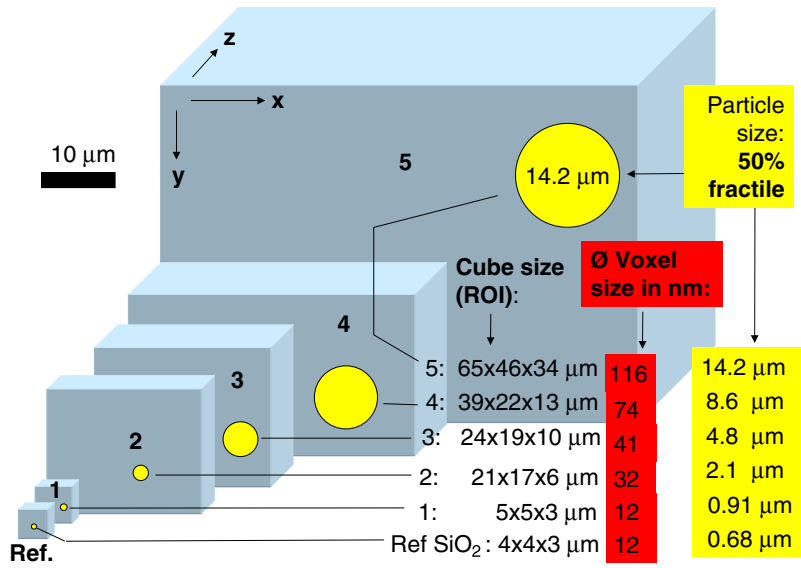

Fig. 4. Visualization of the relative size of sample volumes (ROI cropped from the raw data) for grain size fractions 1 through 5 and for an $\mathrm{SiO}_{2}$ reference sample. The yellow spheres represent the particle size of the $50 \%$ fractile of each powder fraction as obtained with laser diffractometry. of individual particles is a difficult task that impedes the application of any particle analysis method. These problems are briefly illustrated in the following section for 2D microscopy with SEM. Subsequently, the potential of FIB-nt for accurate descriptions of individual objects within these complex granular structures is described at a qualitative level. The precise object recognition is then the basis for further statistical particle analysis. The corresponding computational procedures are particularly discussed in part II of this article. ${ }^{15}$

\section{(1) Precise Object Recognition from Complex Granular Textures}

Figure 6 shows an example of agglomerated particles from fraction 3, captured in the ESEM after ultrasonic treatment (5 min) and filtration from a very dilute suspension in alcohol. The formation of such agglomerates is considered to be the product of hydration processes that took place when the powder was stored under ambient conditions. With the automated particle analysis in ESEM, where segmentation is based on simple thresholding, the object in Fig. 6 was treated as a single entity. However, visual inspection clearly shows that the object is a composite of distinct parts and that some of these pieces $(1,2,3 \ldots)$ should be

Table III. Summary of 3D-data Volumes Acquired with FIB-nt from different Grain Size Fractions

\begin{tabular}{|c|c|c|c|c|c|c|c|c|c|c|c|c|c|c|c|}
\hline \multirow{2}{*}{$\begin{array}{l}\text { Grain size } \\
\text { fraction }\end{array}$} & \multirow[b]{2}{*}{ Magnification $\times 10^{3}$} & \multicolumn{4}{|c|}{ Voxel dimensions ${ }^{\dagger}$} & \multicolumn{4}{|c|}{ Voxel matrix } & \multicolumn{4}{|c|}{ Dimensions of data volume } & \multicolumn{2}{|c|}{ Particles } \\
\hline & & $X(\mathrm{~nm})$ & $Y(\mathrm{~nm})$ & $Z(\mathrm{~nm})$ & Øsize (nm) & $X(-)$ & $Y(-)$ & $Z(-)$ & Total $\left(10^{6}\right)$ & $X(\mu \mathrm{m})$ & $Y(\mu \mathrm{m})$ & $Z(\mu \mathrm{m})$ & Total $\left(\mu \mathrm{m}^{3}\right)$ & Total $^{\ddagger}$ & Inside \\
\hline 1 & 35 & 8.5 & 10.8 & 17 & 12 & 607 & 505 & 170 & 52.1 & 5.2 & 5.4 & 2.9 & 81 & 1659 & 937 \\
\hline 2 & 10 & 29.7 & 37.7 & 30 & 32 & 690 & 439 & 183 & 55.4 & 20.5 & 16.5 & 5.5 & 1860 & 3767 & 243 \\
\hline 3 & 10 & 29.7 & 37.7 & 60 & 41 & 793 & 509 & 159 & 64.2 & 23.5 & 19.2 & 9.5 & 4307 & 2236 & 1404 \\
\hline 4 & 5 & 59.4 & 75.4 & 90 & 74 & 654 & 291 & 145 & 27.6 & 38.8 & 21.9 & 13.1 & 11112 & 1236 & 786 \\
\hline 5 & 3.5 & 84.9 & 107.7 & 170 & 116 & 769 & 429 & 199 & 65.7 & 65.3 & 46.2 & 33.8 & 101992 & 1953 & 1238 \\
\hline
\end{tabular}

${ }^{\dagger}$ Voxels are not isometric: the average voxel size (Øsize) is defined as the edge length of a cube of equivalent volume. ${ }^{\ddagger}$ Total includes particles that are truncated at the volume boundary. FIB-nt, focused ion beam-nanotomography.

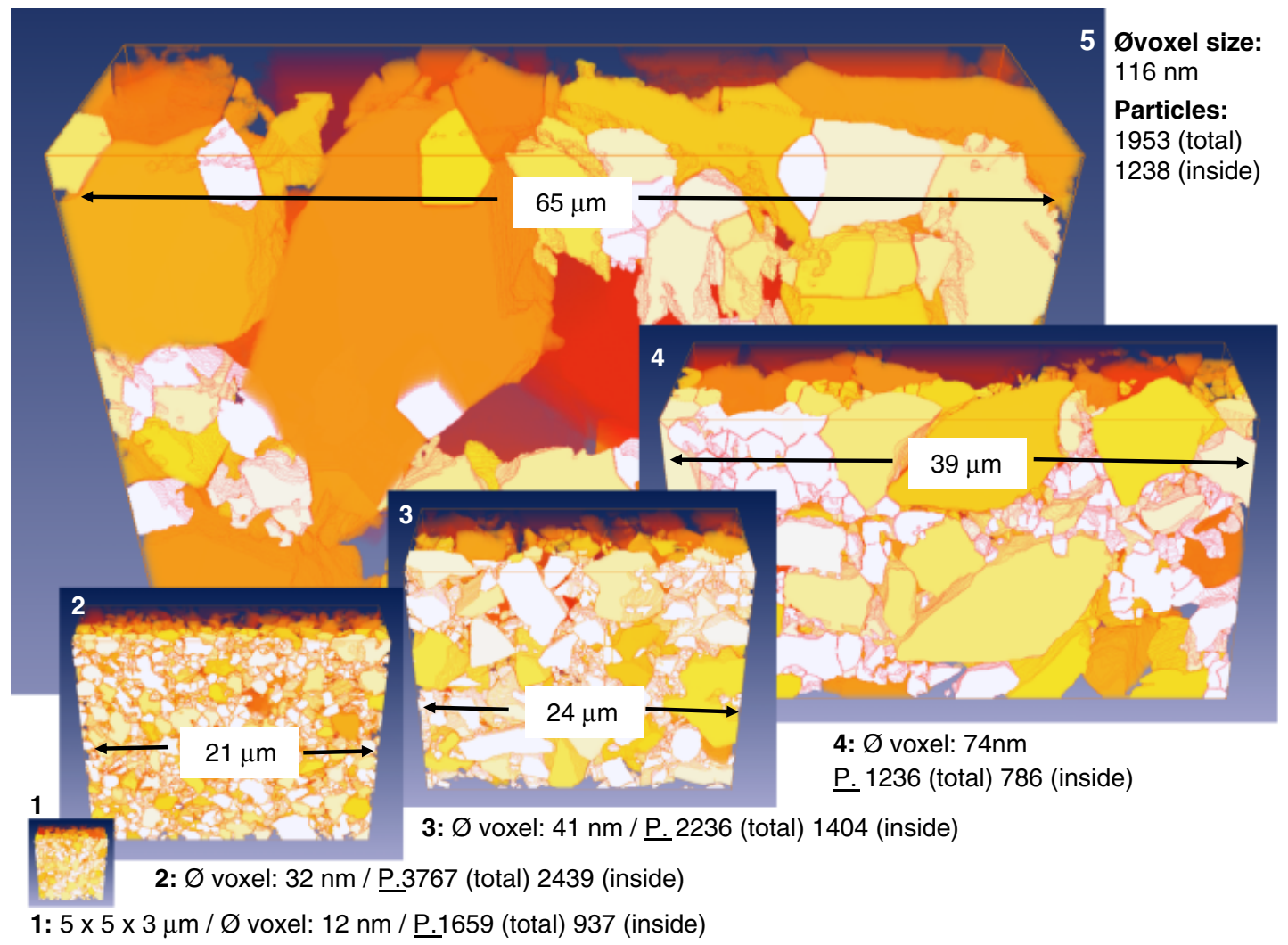

Fig. 5. Scale models for visualization of the relative cube dimensions from grain size fractions 1 to 5 . Each particle is labeled with a distinct color value. 


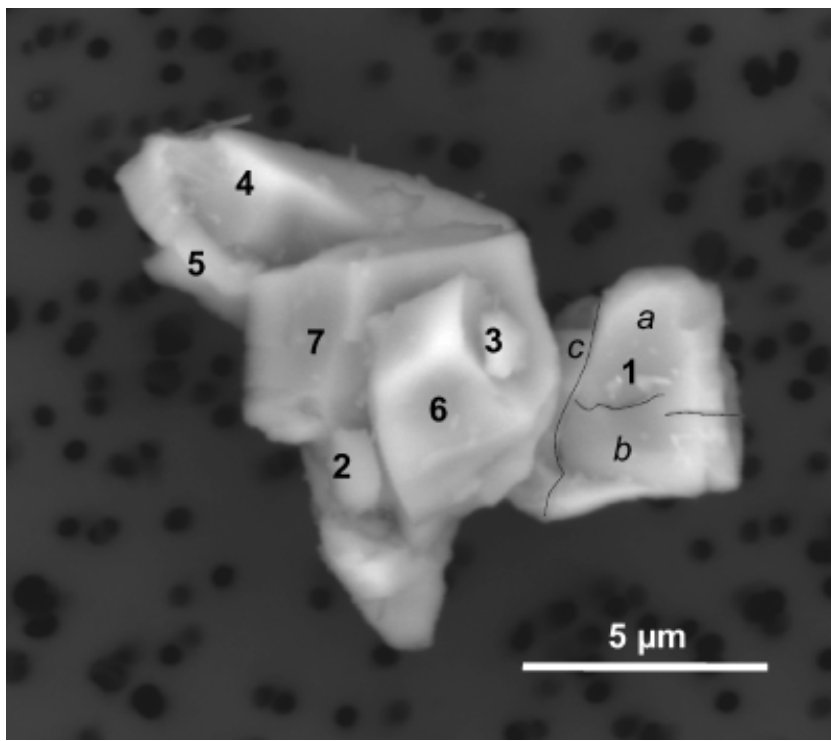

Fig. 6. Secondary electron image (ESEM) of a complex cement particle after filtration from a very dilute suspension in alcohol from grain size fraction 3. The image documents the tendency of cement particles to form agglomerates. Particle no. 1 has fragmented into several subgrains (a, b, c) along internal microcracks. Agglomeration and fragmentation represent major problems for the identification of individual objects, which impedes any particle analysis approach.

classified as individual particles. A further complication could be observed in particle no. 1 , which is subdivided into several fragments ( $a, b$, and $c)$ due to internal microcracks. This fragmentation can be attributed to the thermal (rapid cooling) and mechanical treatment (milling) of the powder. Processes such as cement hydration are kinetically controlled by the surface area that is accessible to the pore solution. Hence, grain fragmentation may need to be taken into account when modeling such processes. From the example in Fig. 6, it becomes evident that 2D information from an SEM projection is insufficient for the localization of the subgrain boundaries. For this purpose, high-resolution $3 \mathrm{D}$-information is required.

The potential of FIB-nt to resolve the details of multi-grain features below the $\mu \mathrm{m}$-scale is documented for two examples in Fig. 7. Both examples originate from selected areas that were enlarged from the image stack of sample 3. The left series of images represents agglomerated particles, which form a dense intergrowth. The right series shows a strongly fragmented domain, where a larger particle has disintegrated into numerous small subgrains. FIB analyses revealed 19 individual objects in the agglomerate (bottom left) and 37 small subgrains in the fragmented domain (bottom right). In both cases, 2D microscopy would not be capable of resolving the numerous subgrains and would therefore treat the entire features as single objects. Also, conventional 3D techniques such as X-ray tomography would not be able to resolve these details. The potential of FIB$\mathrm{nt}$ to detect these small particles is based on the higher quality of 3D data and combination with more sophisticated segmentation techniques; see Munch et al. ${ }^{15}$ Thus, the particular potential of FIB-nt is the reliable identification even of tiny objects from within complex granular textures. This is a prerequisite for accurate statistical description of PSD and particle shape.

\section{(2) Particle Shape Analysis: Moments of Inertia}

The predictability of the workability of concrete and cementitious material as a whole is a subject of growing interest with significant practical application. In this context, the proper simulation of cement paste is a challenge that needs to be met. This involves many difficulties, among which the statistical description of particle shapes is of major importance. As noted in the
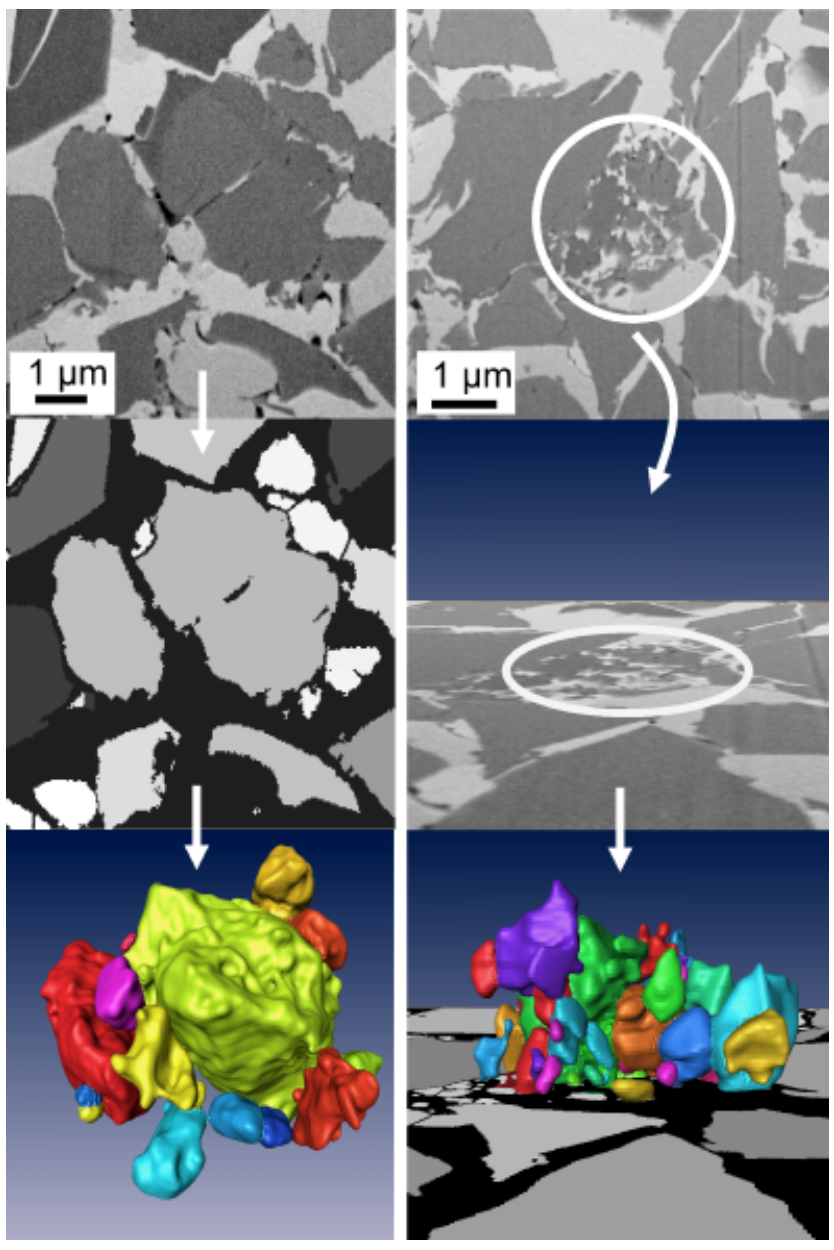

Fig. 7. Two examples illustrating the extraordinary potential of focused ion beam-nanotomography (FIB-nt) to detect individual particles in complex granular textures. The left series of images shows an agglomerate that consists of 19 distinct particles (top: FIB-SEM grayscale image, middle: same image after segmentation, bottom: $3 \mathrm{D}$ reconstruction of the agglomerate). The right series represents a strongly fragmented domain with 37 subgrains (top: FIB-SEM grayscale image, middle: same image tilted, bottom: 3D reconstruction of fragmented domain superimposed on segmented and tilted image). Both agglomeration and fragmentation are frequently observed features in cement powders.

introduction, DPD simulations by Martys ${ }^{3}$ are now at the stage that allows following the motion of particles of complex shapes in a shear flow.

We have shown in the previous sections that FIB-nt is a technique that can provide detailed $3 \mathrm{D}$ characterization of the shape of cement (or any other) particles down to sizes that cannot be performed by other techniques. However, there is practical interest in reducing the extent of experimental work necessary to characterize a given powder before running such simulations. In particular, the question of whether shape factors changes significantly with particle size needs to be addressed.

In this context, the most pertinent parameter to examine versus size for rheology simulations is moment of inertia. For a 3D particle volume with a given mass density distribution $\rho(r)$, the inertia tensor becomes

$$
J=\int_{V} \rho(r)\left(\begin{array}{ccc}
y^{2}+z^{2} & -x y & -x z \\
-y x & x^{2}+z^{2} & -y z \\
-z x & -z y & x^{2}+y^{2}
\end{array}\right) \mathrm{d}^{3} r
$$

The symmetric tensor exhibits real eigenvalues, wherefore an orthogonal main axis system composed of the eigenvectors can be defined as a rough particle shape estimator yielding an inertia 

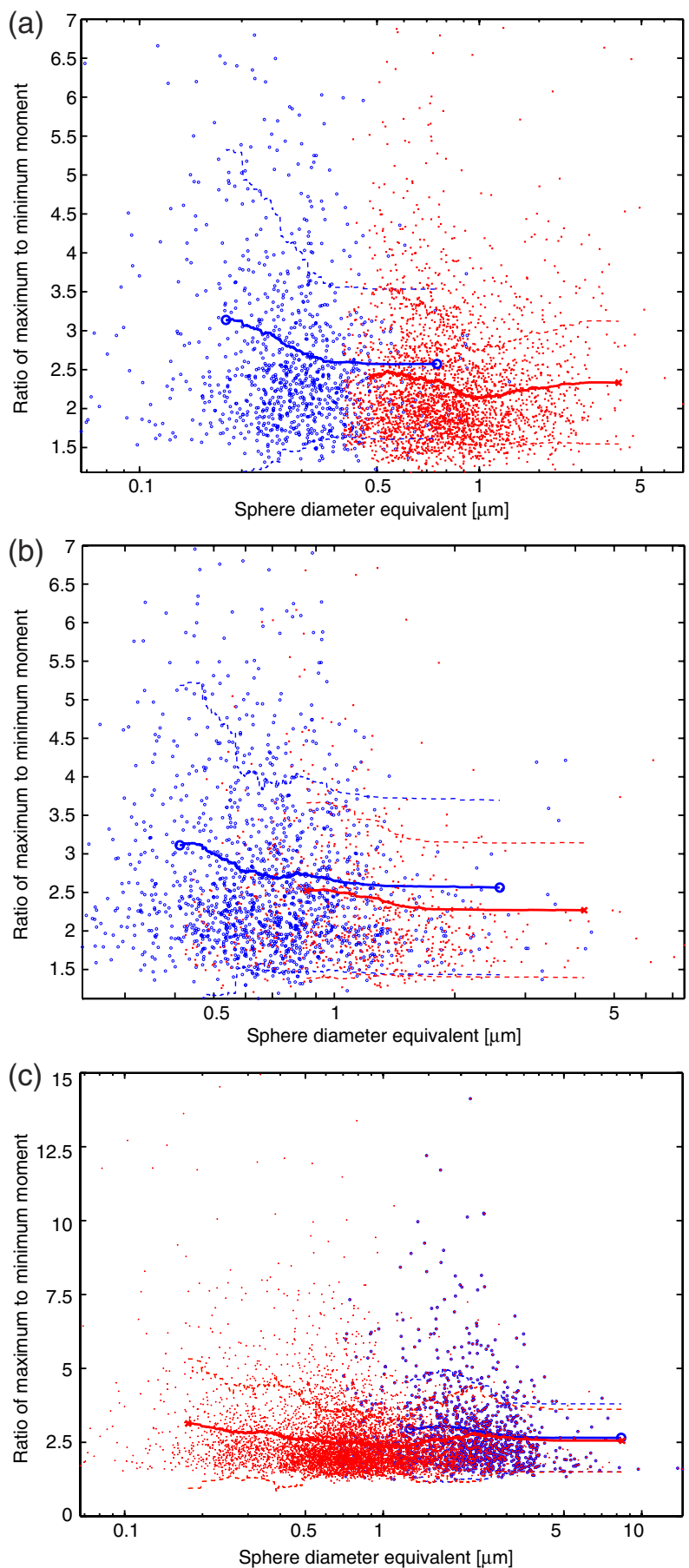

Fig. 8. Particle shape analysis for cement grain size fractions 1 to 5 showing the aspect ratio $r_{\min \max }$ of the minimal and maximal moment of inertia versus particle size (i.e., volume-equivalent particle diameter). For the mean ratio of each size class, 200 particles are averaged, respectively. In (a) blue line, blue dashed lines, and blue circles represent mean aspect ratio, standard deviation bands $\sigma_{r}$, and data points of fraction 1 , red lines and crosses represent fraction 2. In (b): blue lines and circles $=$ fraction 3 , red lines, and crosses $=$ fraction 4 . In (c): blue lines and circles $=$ fraction 5 , red lines, and crosses $=$ all fractions.

tensor of $J=\operatorname{diag}\left(J_{1}, J_{2}, J_{3}\right)$, as is commonly known. The resulting three main moments of inertia $J_{1}, J_{2}, J_{3}$ comprise the minimal as well as the maximal moment $J_{\min }$ and $J_{\max }$. Their aspect ratio $r_{\min \max }=J_{\max } / J_{\min }$ is considered to be a first measure for shape estimation.

For this study, the moments of inertia were determined for all particles in grain size fractions 1 to 5. Particles that are affected by truncation at the cube boundaries were neglected. Figure $8(\mathrm{a}-\mathrm{c})$ shows the aspect ratio $r_{\min \max }$ versus the particle size (i.e., the volume-equivalent spherical diameter). For all five fractions, the average $r_{\min \max }$ was within a relatively narrow band of around $2.7 \pm 0.5$. This implies that the aspect ratio $r_{\min \max }$ did not seem to be size dependent. With respect to chemical and mineralogical variations, it is well known that there is a sizedependent fractionation of phase contents (e.g., the relative enrichment of gypsum in the fine fraction of OPC). But our results indicated that the average aspect ratio $r_{\min \max }$ over the entire size range was not noticeably affected by the phase-fractionation phenomena. For each fraction, however, there was a trend of decreasing $r_{\min \max }$ with increasing particle size $\left(r_{\min \max }\right.$ of fractions 1 and 2 decreased from 3.2 to 2.6 ; from 2.6 to 2.4 in fractions 3 and 4; and from 3.1 to 2.7 in fraction 5). As this trend was observed for all fractions, systematic reasons may be expected. A possible explanation is the fact that particles are more likely to be affected by boundary truncation if their aspect ratio $r_{\min \max }$ is large. Moreover, the probability to become truncated by the boundary planes rises for increasing particle size. Combined, large particles with high aspect ratio $r_{\min \max }$ indicating a weak isometry are more likely to undergo boundary truncation and thus to be withdrawn from the statistics. Consequently, the mean $r_{\min \max }$ of the cement particles to the left sides of the particle diameter bands (values around 3) are more reliable than $r_{\min \max }$ to the right sides. In addition, the standard deviations $\sigma_{r}$ of $r_{\min \max }$ (dashed lines) were fairly large for all fractions. This implies that working with a set of ellipsoids of just one single average size may produce misleading results for rheological models. On average, the ratio $r_{\min \max }$ and the standard deviation $\sigma_{r}$ exhibited similar features and comparable characteristics for all 5 particle fractions. Consequently, a dramatic reduction in the degree of characterization of particle shape is justified before simulating the flow of cement suspensions.

\section{(3) Topological Characterization of Granular Textures with FIB-nt-An Outlook}

As discussed in the introduction, there are numerous microstructural features that have so far hardly been characterized with conventional experimental techniques in granular materials and even more so when agglomerated: the number of contacts, contact curvatures, or percolation of networked particle structures. High-resolution 3D microscopy with FIB-nt provides new possibilities to describe these complex microstructural features precisely .

Figure 9 shows an example of the reconstruction of the interfaces between neighboring particles in a grain-supported texture. The upper left cube represents a selected area from the cube of sample 3, which contains approximately 150 particles. The red, curved areas (top right) show the contact surfaces between the particles. In order to illustrate the relationship of the contact surfaces with the granular textures, 30 particles were selected (bottom left) and superimposed on the contact surfaces (bottom right). The example illustrates the potential of FIB-nt to describe topological details of particle interfaces. This is of particular interest, as macroscopic properties of granular materials are strongly related to the microscopic interface characteristics. Future research activities with FIB-nt in the field of granular materials will focus on a quantitative description of the interface topology and agglomerated features and on an elaboration of their quantitative relationship with macroscopic materials properties, such as mechanical properties (strength, elasticity) in solid granular materials or flow properties in loose particle systems by means of cryo-fixation techniques (see Table I).

\section{Conclusions}

FIB-nt is a novel high-resolution 3D-microscopy method. Because of the automated drift-correction procedure, a reproducible interlayer spacing of $15 \mathrm{~nm}$ can be achieved. In contrast, conventional FIB serial sectioning without drift correction will be affected by mechanical and electronic instabilities. Therefore, at high resolutions, only the drift-corrected FIB-nt can reveal 


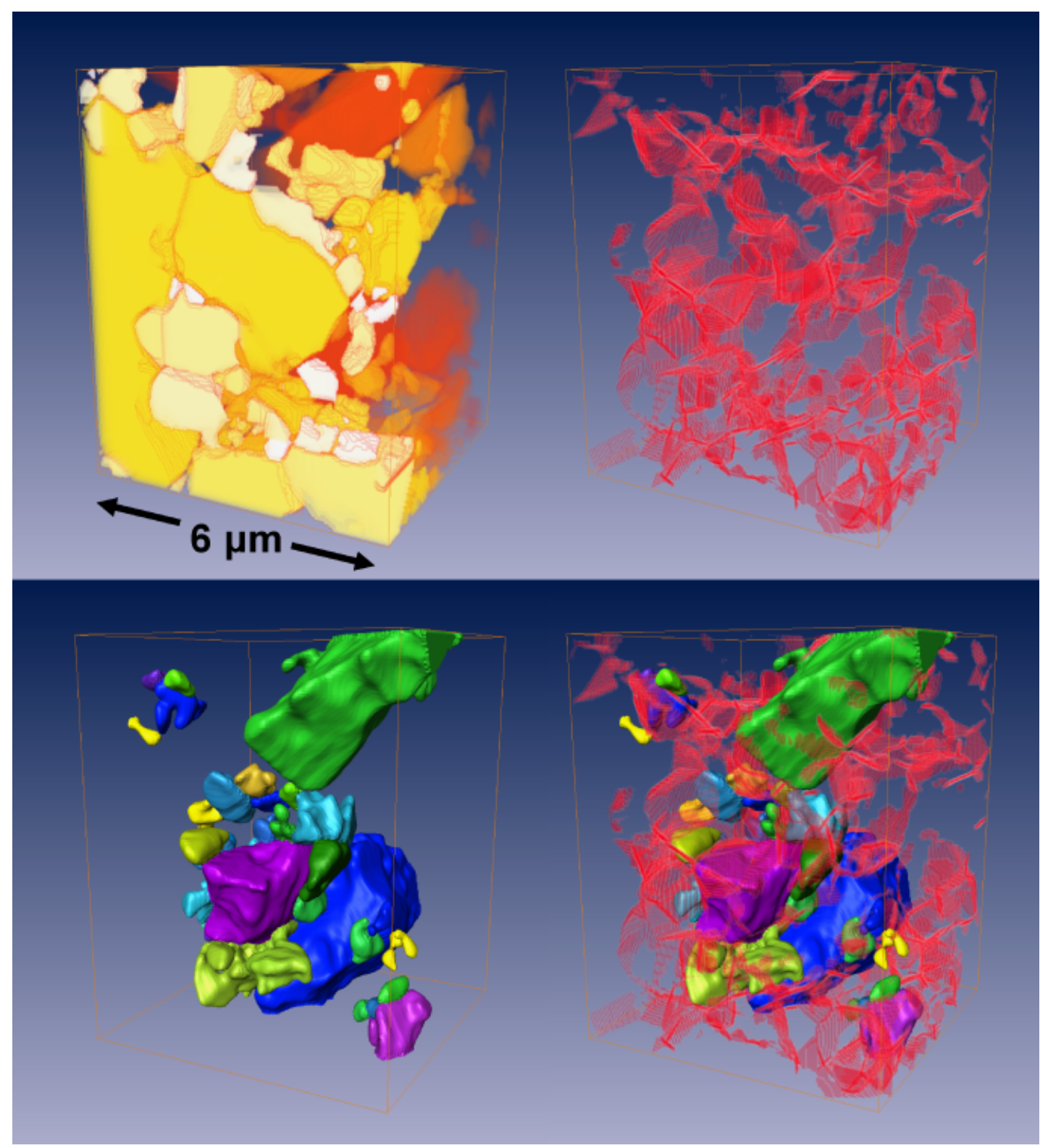

Fig.9. Topological characterization of particle-particle interfaces based on 3D analysis with focused ion beam-nanotomography: All four images represent the same sample locality (size: $6 \times 8 \times 6 \mu \mathrm{m} /$ voxel size: $30 \mathrm{~nm} \times 38 \mathrm{~nm} \times 60 \mathrm{~nm}$ ). Top left: Selected area from grain size fraction 3 with 150 particles. Top right: Interfaces of neighboring particles. Bottom left: Selection of 30 particles. Bottom right: Superposition of interfaces with selected particles.

accurate $3 \mathrm{D}$ data that are suitable for quantitative microstructure analysis.

In this study, the potential of FIB-nt for detailed characterization of particles and granular textures has been demonstrated convincingly. Currently, there is no other method that can reveal morphological particle information at $15 \mathrm{~nm}$ resolution from within relatively thick samples. Hence, even from dense and complicated granular textures, the grain boundary and surface geometry of the individual objects can be reconstructed and used for statistical particle analysis. From the shape analysis of the investigated PC, it follows that the average moment of inertia was similar for all five grain size fractions, i.e., no size dependency was observed. The average aspect ratio of maximum to minimum principal axis was close to 3 , although standard deviations were very large. Furthermore, the surfaces of interparticle contacts could be extracted from within dense granular textures. This provides the possibility to quantify number of contacts and contact curvatures.

For further statistical analysis, particularly for determination of the PSD, the data have to be corrected for truncation effects at the boundary, which preferentially affect the larger and non- isometrical particles. The stereological correction of the boundary truncation is the issue of a second, forthcoming publication about particle analysis based on FIB-nt. ${ }^{15}$ Further exploitation of the FIB-nt method for particulate systems, both underway and planned, involves comparing PSD with those obtained by other techniques, computation of coordination numbers and comparison with predictive rheological models for simplified geometries, and incorporation of irregular particle shapes into hydration models.

\section{References}

${ }^{1}$ K. Liffman, M. Nguyen, G. Metcalfe, and P. Cleary, "Forces in Piles of Granular Material: An Analytic and 3D DEM Study," Granular Matter, 3, 165-76 (2001).

${ }^{2}$ B. Peters and A. Dziugys, "Numerical Simulation of the Motion of Granular Material Using Object-Oriented Techniques," Comput. Meth. Appl. Mech. Eng., 191, 1983-2007 (2002).

${ }^{3}$ N. S. Martys, "Study of a Dissipative Particle Dynamics Based Approach for Modeling Suspensions," J. Rheol., 49 [2] 401-24 (2005).

${ }^{4}$ D. M. Scott, "Characterizing Particle Characterization," Part. Part. Syst. Char., 20, 305-10 (2003). 
${ }^{5}$ R. Clift, "Powder Technology and Particle Science," Powder Technol., 88, 3359 (1996).

${ }^{6}$ R. M. German, Particle Packing Characteristics. Metal Powder Industries Federation, Princeton, 1989.

${ }^{7}$ E. J. Garboczi and J. W. Bullard, "Shape Analysis of a Reference Cement," Cement Concrete Res., 34, 1933-7 (2004).

${ }^{8}$ B. J. Inkson, M. Mulvihill, and G. Möbus, "3D Determination of Grain Shape in a FeAl-Based Nanocomposite by 3D FIB Tomography," Scripta Mater., 45, 753-8 (2001).

${ }^{9}$ B. J. Inkson, T. Steer, G. Möbus, and T. Wagner, "Subsurface Nanoindentation Deformation of Cu-Al Multilayers Mapped in 3D by Focused Ion Beam Microscopy," J. Microsc., 201 [2] 256-69 (2001).

${ }^{10}$ FEI-Company, Auto Slice and View ${ }^{\mathrm{TM}}$ for Dual Beam FIB, 2004

${ }^{11}$ L. Holzer, F. Indutnyi, Ph. Gasser, B. Münch, and M. Wegmann, "3D Analysis of Porous $\mathrm{BaTiO}_{3}$ Ceramics using FIB Nanotomography," J. Microsc., 216 [1] 84-95 (2004)

${ }^{12}$ K. L. Willis, A. B. Abell, and D. A. Lange, "Image-Based Characterization of Cement Pore Structure using Wood's Metal Intrusion," Cement Concrete Res., 28 [12] 1695-70 (1998).
${ }^{13}$ A. K. Crumbie, "SEM Microstructural Studies of Cementitious Materials: Sample Preparation of Polished Sections and Microstructural Observations with Backscattered Images - Artefacts and Practical Considerations"; pp. 320-41 in Proceedings of the 23rd International Conference on Cement Microscopy, Edited by L. Jany and A. Nisperos. Int. Cement. Micr. Association (ICMA), Albuquerque, 2001.

${ }^{14}$ FEI-Company, AutoScript, Technical Note PN 25564-C. 2000.

${ }^{15}$ B. Münch, L. Holzer, R. J. Flatt, and Ph. Gasser, "FIB-Nanotomography of Particulate Systems - Part II: Particle Recognition and Correction of Boundary Truncation. J. Am Ceram. Soc., 89 [8] 2586-2595 (2006).

${ }^{16}$ A. Tewari and A. M. Gokhale, "Estimation of Three-Dimensional Grain Size Distribution from Microstructural Serial Sections," Mater. Character., 46, 329-35 (2001).

${ }^{17}$ N. Chawla, V. V. Ganesh, and B. Wunsch, "Three-Dimensional (3D) Microstructure Visualization and Finite Element Modeling of the Mechanical Behavior of $\mathrm{SiC}$ Particle Reinforced Aluminum Composites," Scripta Mater., 51 [2] 161-5 (2004).

${ }^{18}$ H. Singh and A. M. Gokhale, "Visualization of Three-Dimensional Microstructures," Mater. Character., 54, 21-9 (2005). 